\title{
QASIM AMIN Y JOHN STUART MILL: LAS RAZONES DE LA ESCLAVITUD FEMENINA
}

\author{
ENCARNACIÓN RUIZ CALLEJÓN \\ Universidad de Granada
}

Recibido: $31 / 08 / 2015$

Aceptado: 16/09/2015

\section{Resumen}

Qasim Amin (1863/5-1908) no fue el primer autor árabe que en el contexto del islam contemporáneo escribió y militó a favor de la liberación de las mujeres, pero ha sido considerado el primer teórico y el antecedente por excelencia del movimiento feminista egipcio. El objetivo de este artículo es caracterizar el feminismo del autor en relación a su diagnóstico sobre la crisis de mundo árabe. Me centro para ello en las siguientes cuestiones: las tesis de Amin sobre la esclavitud femenina y la articulación entre sus causas y el reformismo del autor atendiendo a los criterios que él mismo señala: la libertad y el interés común. Para caracterizar mejor su posición, considero también las tesis de Amin en relación a algunas de las críticas y propuestas de Stuart Mill sobre el sometimiento del mujer, al ser Mill una de sus fuentes de inspiración.

Palabras clave: filosofía del siglo XIX - liberación de las mujeres- reformismo árabe contemporáneo.

\begin{abstract}
Qasim Amin (1863/5-1908) was not the first Arab author in the context of contemporary Islam who wrote and campaigned for the liberation of women, but has been considered the first theorist and the antecedent par excellence in the history of the Egyptian feminist movement. The aim of this paper is to characterize the author feminism in relation to its assessment of the crisis in the Arab world. In particular, I focus on the following issues: Amin's thesis on female slavery and the relationship between its causes and reformism of the author, according to the criteria he himself points out: freedom and common interest. To further characterize his position, I also consider
\end{abstract}


Amin's thesis in relation to some of the criticisms and proposals of Stuart Mill on the subjugation of women, one of his sources of inspiration.

Keywords: Arab contemporary reformism- liberation of the women - 19th Century Philosophy. 


\section{La reforma empieza con la emancipación de las mujeres ${ }^{1}$}

El interés de los estudiosos sobre el reformismo árabe se ha centrado en el papel de la religión en la larga crisis del mundo árabe, pero poco a poco se van cuestionando tanto categorías como «modernidad», «tradición», «crisis del mundo árabe», «renacimiento» (Nahda), etc., como la unidad y uniformidad del propio movimiento reformista, su origen y agentes, el marco espacio-temporal e incluso las temáticas ${ }^{2}$. De hecho, uno de los aspectos más significativos de su discurso fue la emancipación de las mujeres, un aspecto que está siendo investigado con más intensidad hoy. Las primeras fases del debate habrían tenido lugar en la Gran Siria en donde la «cuestión de las mujeres» (Masalat al-Nisa) aparece como un componente intrínseco al programa de regeneración. Prendió luego en Egipto de la mano de intelectuales exiliados, escritores y periodistas. Esta primera fase culminaría con la reflexión de Qasim Amin ${ }^{3}$, lo cual no niega la importancia de este, pero modifica sustancialmente una de las primeras valoraciones: sin él el siglo XIX habría acabado sin que la cuestión de la emancipación de las mujeres adquiriese una dimensión social (Zachs 18-19). Mientras varios de los reformistas egipcios consideraron en su

1. Este trabajo se inscribe en el proyecto de investigación del Grupo de Investigación financiado por la Junta de Andalucía: «Antropología y Filosofía» (SEJ-126).

2. Ver: Abdulrazzak Patel. The Arab Nahdah. The making of the Intellectual and Humanist Movement; Ilham Khuri-Makdisi, The Eastern Mediterranean and the Making of Global Radicalism, 1860-1914; Charles Saint-Prot. La tradición islámica de la reforma.

3. Antes de la primera revista dirigida y escrita por mujeres, aparecida en 1892 , un pequeño número debatía sus inquietudes sociales y políticas en revistas dirigidas por hombres. Y cinco años antes de La liberación de la mujer, autoras como Zaynab Fawwaz y Hanna Kawrani abordaban el tema del sufragio. Cuando Amin publicó su obra, ya existían más de seis revistas debatiendo los mismos problemas (Mazid 48). Sobre la historia del movimiento feminista egipcio puede consultarse una obra pionera y ya un clásico en castellano: Caridad Ruiz de Almodóvar. Historia del movimiento feminista egipcio. Para una panorámica resumida con una síntesis del logro de derechos, ver: Nooh Ramadan, Anfänge der Frauenbewegung und Genderdiskussion in Ägypten. Am Beispiel von Qasim Amin und Hoda Sha'arawi als Begründer, 4-12. Beth Baron en Egypt as a Woman. Nationalism, Gender and Politics analiza, entre otras cosas, cómo el discurso y las representaciones sobre Egipto que se dan 1919 y los años 40 están repletos de una imaginería femenina. 
agenda la necesidad de cambios en la situación de las mujeres, Amin dedicó a ello la mayor parte de sus energías y unió el éxito del nacionalismo a la abolición de prácticas del patriarcado de la clase alta poniendo de relieve el atraso de la institución del harén. Por la misma época estaba floreciendo en Egipto una variada literatura hecha por mujeres, pero la sociedad árabe instruida y los orientalistas dieron preferencia a las obras escritas por hombres (Abisaab 2).

Qasim Amin procedía de una familia aristocrática otomana. Tras acabar derecho ocupó un alto cargo en el ejército del Pasha Ismail (1830-1895). Fue uno de los 37 estudiantes becados en 1881 por el gobierno para completar su formación durante cuatro años en la Universidad de Montpellier. En esta época trabajó también en la revista Al-Urwa al-Wuzqa (El lazo indisoluble) editada por Al-Afgani (1838-1897) y Muhammad Abdu (1839-1905), dos de los principales artífices del reformismo árabe contemporáneo, y fue el traductor de Abdu. Al volver a Egipto contrajo matrimonio emparentando con una familia egipcia aristocrática y desarrollando su carrera profesional en puestos de relevancia.

Suele recordarse que Amin no se había planteado inicialmente defender la emancipación de las mujeres. En 1893 Charles-François-Marie, IX Duque de Harcourt (1835-1895), se refería de forma despectiva al país y culpaba al islam de la situación de las mujeres en L'Egypte et les Egyptiens. Amin le respondió en Les Egyptiens. Response a M. Le Duc d'Harcourt (1894) cuestionando su representación estereotipada del musulmán ${ }^{4}$ y defendiendo la situación de la mujer egipcia. Sin embargo, años después le confió a un amigo que no estaba satisfecho con tal defensa ${ }^{5}$. De hecho en 1899 publicó La liberación de la mujer ${ }^{6}$, dirigido a sus compatriotas y con un objetivo bien distinto. En 1900, y como respuesta a las críticas que había recibido, publica $L a$ nueva mujer, dedicada a Saad Zaghlul (1859-1927), uno de los padres de la nación egipcia. Ambos textos generaron una gran reacción en contra (Ruiz de

4. «Islam encourages pleasures and enjoyment of all that we harbor in our hearts of emotions and yearnings, except for gluttony and voraciousness. He [D'Harcourt] spoke aplenty of lust, obscenity, and degeneration without ever telling us where we saw such things» (Massad 56).

5. «I could not shake from my mind one important subject which we had debated. I am referring, of course, to the problem of the Egyptian woman. Up until that time, I had been totally unaware that something might be wrong with our family patterns» (Mazid 43).

6. Tahrir al-mara a veces aparece traducido como: La liberación de las mujeres. Ocurre, por ejemplo, en la traducción al inglés. Pero «mara» es singular: mujer. Debe ser por tanto: La liberación de la mujer. Hecha esta aclaración, para no crear confusión en las referencias bibliográficas, mantengo sin embargo los títulos de las obras consultadas. 
Almodóvar 58-59), pero también un intenso debate: la primera obra dio lugar a más de una treintena de publicaciones de hombres y mujeres debatiendo la cuestión del velo. Para algunos estudiosos, lo que motivó la intensa reacción en contra fueron los argumentos pro-europeos y orientalistas del autor, consecuencia de su trayectoria académica y profesional ${ }^{7}$, porque parecían incluso justificar un Egipto sometido en un momento en el que emergía precisamente el nacionalismo. Para otros, la obra de Amin continúa el proyecto de Abdu: intentar combinar modernidad e islam (Pacheco 82). También ha sido considerado «el pionero de una línea de análisis liberal» y no del reformismo, que atribuía la situación de la mujer a una errónea interpretación del islam (Azaola 162). Ha sido considerado un secularista al final de su producción y por ello un antecedente del cristiano sirio Farah Antun (1874-1922), si bien para este el secularismo será el punto de partida para transformar el mundo árabo-islámico (Reid 1975, 94). Celia Amorós se refiere a Qasim Amin como el «Stuart Mill egipcio» siendo su feminismo funcional a la vez que sustantivo ${ }^{8}$. Durante la estancia en Francia, Qasim Amin entró en contacto con las obras de los autores en los que más tarde se inspiraría, como es el caso de $\mathrm{El}$ sometimiento de la mujer de John Stuart Mill, compuesta entre 1860 y 1861 y publicada en 1869. Entre 1865 y 1868 Mill fue miembro del Parlamento, presentando en 1866 con Henry Fawcett una petición firmada por 1499 mujeres para cambiar la ley del sufragio: «el único servicio de real importancia que presté como miembro del Parlamento» (Miguel Álvarez 206) ${ }^{9}$, dirá más tarde.

7. Había recibido una educación basada en un curriculum europeizado tanto en la escuela primaria de Alejandría como en la Cairo Preparatory School y en su formación de posgrado. En cuanto a los cargos que ocupó: «he became a part of the empire's civil servant class (...) In 1885 he became a juror in the Mixed Courts, a judiciary system saturated in foreign western influence. The Mixed Courts, created 10 years before Amin's appointment, were based on a combination of the Napoleonic judicial system and Islamic law. It had foreign judicators from England, Austria, Germany, and France, and it was these foreign powers who nominated jurists for judicial posts. Amin continued to have a successful career in these foreign-run judicial offices. In 1887, he entered the predominately western-run Egyptian office of the Government Division of Legal Affairs. By 1889, he was appointed one of the National Courts' Egyptian judges. Given his extensive training in imperial schools and institutions, it was no wonder that Orientalist dogma found its way into Amin's worldview» (Mazid 53).

8. «reconoce a las mujeres el derecho a cultivar su inteligencia y sus dotes naturales, así como a disfrutar de la felicidad, en primer lugar, para sí mismas, y, aunque se enfatice constantemente, en segundo lugar, para que ese desarrollo sirva al bienestar de la familia» $(2009,272)$.

9. Su padre, James Mill, había negado el voto a las mujeres porque los intereses de estas estarían ya incluidos en los de sus padres o maridos (Miraut Martín 104). Por otro lado, en la época de su actividad parlamentaria nuestro autor se encontraba «muy sensibilizado (...) por las circunstancias personales que le había tocado vivir con su esposa,

Feminismo/s 26, diciembre 2015, pp. 57-81 
En 1868 se instaló cerca de Aviñón, donde estaba enterrada su esposa Harriet, y allí ultimó el texto para publicarlo (Rossi 22). Entre 1831 y principios del año siguiente habían escrito, cada uno para el otro, ensayos en los que abordaban los temas del matrimonio y el divorcio y, en general, la situación de la mujer, y que pueden considerarse el germen de la obra. El objetivo de este artículo no es establecer una comparación sistemática entre Qasim Amin y John Stuart Mill, pero para caracterizar mejor la posición del primero señalo aquellos aspectos que aborda Amin y la posición de Stuart Mill al respeto, especialmente en El sometimiento de la mujer.

\section{La esclavitud de las mujeres}

\subsection{El velo y la reclusión}

Según Amin, los musulmanes adoptaron el velo ${ }^{10}$ de otras culturas e hicieron de él un atuendo religioso contrario al espíritu del islam (2000a, 37). El Corán (XXIV, 30-31) ${ }^{11}$ habla en todo caso de recato, es ambiguo el sentido de las aleyas, pero no prescribe el velo integral. Las citadas aleyas permiten enseñar partes del cuerpo a extraños pero no especifican en qué situaciones. Y no hay acuerdo entre los intérpretes, los eruditos y los líderes religiosos. Un hadiz relata que Aisha, la esposa preferida del Profeta, contaba que este le había dicho a Asma, hija también de Abu Bakr, que solo mostrase su cara y sus manos. Amin concluye que son las desventajas del velo en la época la razón por la que la sharía permitía a las mujeres descubrir la cara y las palmas, paliando así una costumbre restrictiva y no originaria del islam.

desafiando primero el ambiente puritano de la época y embebido después por la frustración que le produjo la muerte de su ser más querido» (Miraut Martín 108).

10. El propósito de este trabajo no es llevar a cabo un análisis de cuestiones como la del velo, el matrimonio, la poligamia, etc., en el mundo árabo-islámico o en la ley islámica, sino caracterizar la posición de Qasim Amin. Sobre la cuestión del velo, algunos de los temas que se mencionan en Amin y otros afines, pueden consultarse por ejemplo los trabajos que aluden a las fuentes religiosas, de: Carmelo Beltrán, «Regulaciones pacíficas de género en el Corán», y Katjia Torres Calzado y Juan Antonio Pacheco Paniagua (eds), Disquisiciones sobre el velo islámico.

11. «Di a los creyentes que bajen la vista con recato y que sean castos [lit: que custodien sus partes pudendas] (...). Y di a las creyentes que bajen la vista con recato, que sean castas [lit: que custodien sus partes pudendas] y no muestren más adorno que los que están a la vista, que cubran su escote con el chal y no exhiban sus adornos sino a sus maridos, a sus padres, a sus suegros, a sus propios hijos, a sus hijastros, a sus hermanos, a sus sobrinos carnales, a sus mujeres, a sus esclavas, a sus criado varones fríos [lit: ancianos y eunucos], a los niños que no saben aún de las partes femeninas. Que no batan ellas con los pies [con las ajorcas] de modo que no se descubran sus adornos ocultos $(\ldots) »$. 
Amin afirma que el velo, tal y como fue luego impuesto, impide la igualdad entre hombres y mujeres, reconocida por el islam, porque no es posible ninguna acción legal si la identidad del sujeto, como es el caso de la mujer velada o escondida tras una cortina, no puede comprobarse. Y el recurso a testigos que ratifiquen su identidad favorece más bien el engaño. Un contrato como el del matrimonio se firma a ciegas y con alguien que no se ha visto jamás ${ }^{12}$. Además, Dios no dividió el mundo en dos partes (Amin 2000a, 40). Todas las variedades integrales son una forma de esclavitud: «Es esclava la mujer que es obligada a cubrirse de tal forma que difícilmente pueda andar (...), respirar e incluso hablar (Amin 2000b, 51). El velo «desvirtúa su apariencia exterior y pierde todo rasgo humano» ${ }^{13}$. En La nueva mujer también el manto de paseo (yilbab) y el hiyab «son el baldón de la ignorancia y la debilidad, marchamos fraudulentos» (Amin 2000b, 52) ${ }^{14}$. La finalidad del velo es el control sexual de todas las mujeres. El velo es «el símbolo de esta ancestral tiranía» (Amin 2000b, 54). Un trozo de tela no garantiza virtud alguna (Amin 2000b, 65). Nunca podrá controlar el pensamiento ni cambiar las inclinaciones (Amin $2000 \mathrm{~b}, 68)$ y, por supuesto, no hay virtud sin libertad. El velo oculta los rasgos que permiten hablar de la belleza con naturalidad y promueve una temprana sexualización (Amin 2000b, 63-64). El anonimato y la homogeneidad que impone pueden utilizarse para lo que se desee. El velo supone modelos muy concretos de hombre y de mujer, siembra la desconfianza y da por supuesto que la mujer es incapaz por sí de ser moral. Si la amenaza son los hombres, a ellos hay que velarlos; si las mujeres son las débiles, también, para no ser contemplados: « $i \mathrm{O}$ es que la idea de libertad es diferente para cada sexo y hay dos tipos de derechos?» (Amin 2000b, 59). Cuando se impone por primera vez, la niña apenas ha recibido una educación básica. A partir de ahí, «sin ver el mundo más que a través de las ventanas (...), sin salir a no ser (...) envuelta en una mortaja, no puede llegar nunca a ser una persona viva, que piensa, que está informada de la vida de los demás y que puede convivir con ellos» (Amin 2000b, 128). El primer paso para la libertad femenina es «la desaparición del velo y la abolición de su uso» (Amin 2000b, 55).

12. «Most people refuse to buy a sheep or a donkey before seeing it, examining it thoroughly, and receiving reassurance about any apparent defects. Yet the same rational people go ahead with a marriage with a carelessness that baffles the mind» (Amin 2000a, 77).

13. No comparto la opinión de Nooh cuando afirma que Qasim Amin no se refiere al atuendo en sí, ni cuando afirma que el autor no pone en cuestión la religión, sino solo el patriarcado (Nooh 14-15).

14. De hecho, dedica el penúltimo capítulo explícitamente al hiyab, siendo el término árabe utilizado este (Amin 2015b, 156).

Feminismo/s 26, diciembre 2015, pp. 57-81 
La reclusión también es contraria al islam, que no exige a los creyentes nada difícil u oneroso (Corán II, 185: «Dios quiere hacéroslo fácil y no difícil»; XXII, 78: «Él os eligió y no os ha impuesto ninguna carga en la religión»). No era un deber religioso en la época del Profeta, sino una forma de distinguir a sus mujeres. Aisha tomó parte activa en la vida religiosa, política y militar de la comunidad. La reclusión no solo mina la salud de la mujer, sino que es una forma de ejecución (Amin 2000a, 60), pero el musulmán tiene a gala que no salga de casa a no ser para llevarla al cementerio (Amin 2000b, 50).

\subsection{La educación y la naturaleza femenina}

Se ha constatado que los copistas añadían al conocido hadiz que proclama la búsqueda del conocimiento como un deber para todo musulmán las palabras: «y para toda musulmana». Francis Robinson (2010) ha explorado los espacios y formas de educación de las mujeres a través del mundo musulmán entre el 1000 y el 1800 y aunque evidentemente existían grandes obstáculos y muchos prejuicios contra la mujer con formación ${ }^{15}$ señala, siguiendo a Porter Berkey (1992), que en El Cairo medieval algunos ulemas educaron a sus hijas y las alentaron para que escuchasen la transmisión del hadiz y recibiesen las iyazas, el documento que certificaba sus conocimientos y las autorizaba para enseñar. Con el permiso del marido, una mujer podía asistir a las clases de un erudito en las que había otros hombres. Los parientes jugarán un papel importante enseñando a las mujeres en $\operatorname{casa}^{16}$. Y ellas mismas serán transmisoras y patrocinadoras del conocimiento. En El Cairo fatimí hubo mujeres que se dedicaron a enseñar a otras en sus hogares y en zawiyas u oratorios sufíes. Aquí y en el Damasco de la época, algunas mujeres tuvieron un respetado papel en la transmisión del hadiz. En familias reales como la timúrida o la $\operatorname{mogol}^{17}$ la primera educación de los niños y niñas dependía de las mujeres

15. «'It is said that a woman who learns [how to] write', went a Mamluk market inspector's manual, 'is like a snake given poison to drink' » (Robinson. 526).

16. Zaynab al-Tukhiyya (m. 1388) aprendió a escribir y memorizó el Corán, estudió obras de jurisprudencia y después de casada continuó estudiando los hadices bajo la guía de su marido. Nana Asmapu (m. 1865), hija de Usman dan Fodio, creador del Sokoto califato de Nigeria, enviaba a los pueblos a mujeres para que buscasen niñas menores de catorce años y mujeres mayores de cuarenta y cuatro para formarse con ella y para que luego, a su vez, formasen a otras, y escribió sobre una gran variedad de temas (Robinson 527-528).

17. In the sixteenth century, Fakhrı of Herat produced a biographical dictionary entirely devoted to the poetesses and learned women of Timurid and early Safavid Iran. Focusing on women from noble and scholarly families, it points to active women's engagement in intellectual life, which in the Timurid period was sometimes in mixed company (...) The Mughal royal household was remarkable for its literary and learned women, 
de edad de la casa, que también en el harén otomano enseñaban en una institución similar a la escuela de palacio. En el Sáhara las mujeres de la familia enseñan a niños y niñas los rudimentos de la lectura y la escritura y la lectura y el conocimiento básico del Corán (Robinson 526-528). Qasim Amin, aborda, sin embargo, la situación mayoritaria: a la mujer se le ha impedido educar y desarrollar su intelecto, reduciéndola a una afectividad no cultivada. Su educación es por ello un objetivo imprescindible; no es un lujo ni puede posponerse $\mathrm{e}^{18}$. La mujer es considerada un ser arrastrado por las pasiones y por ello no apta para la formación ni para la moral ya que ambas requieren ser un sujeto de razón $(2000$ a, 55), como si el ser humano no fuese libre o Dios otorgase dones por error o para no usarlos (2000a, 53; 15). La inferioridad intelectual de la mujer no es una enseñanza coránica ni islámica. La ciencia tampoco establece superioridad o inferioridad alguna a partir de las diferencias físicas entre los sexos. De la naturaleza de las mujeres, como de la de los hombres, aún no podemos saber científicamente nada. Pero incluso existiendo diferencias intelectuales, no es este un argumento para privarlas de libertad: « $i$ o es que entre los varones (...) no hay diferentes niveles de inteligencia?» (Amin 2000b, 59).

En el caso de Stuart Mill, encontramos el mismo argumento, pero se relaciona con su reflexión más extensa sobre el concepto de naturaleza. Del conjunto de características de algo no se desprende ningún criterio normativo $(2002,9)$. Ser lo que somos tampoco es un mérito, porque no se elige (2012, 19). Por tanto, actuar conforme a la naturaleza o seguir la naturaleza debe entenderse como intentar encauzar los bienes que encontramos hacia la justicia y la bondad $(2012,67)$, y en el caso de los instintos perjudiciales, controlarlos con la educación o mediante técnicas como dejarlos morir por falta de uso $(2012,58)$. Dicho esto, lo que se denomina «naturaleza femenina» es un concepto ambiguo y falto de fundamentación. La ciencia no ha determinado

produced in every generation from the emperor Babur's (r. 932-7/1526-30) daughter, Gulbadan Begum (d. 1011/1603), to the emperor Aurangzeb's daughter Zeb al-Nisap (d. 1114/1702). Few, however, were likely to match the range of Nana Asmapu, who wrote in Arabic, Fulfulde and Hausa on: health, women's education, law as it applied to women, women and bori (spirit possession), women as sustainers, the family, history, eschatology, politics, theology and her father's caliphate» (Robinson 527-528).

18. Amin formó parte del grupo que creó la Universidad de El Cairo y apoyó la entrada de la mujer en ella, una universidad que explícitamente se presentaba abierta a todo el que buscase el conocimiento sin importar su nacionalidad o religión. El primer año de su funcionamiento, el año de la muerte de Amin, 31 mujeres estaban en sus aulas (Reid 2002). 
la naturaleza del hombre ni de la mujer ni tampoco la inferioridad de esta ${ }^{19}$. No se ha podido establecer que su cerebro sea menor. Ni siquiera se conoce la relación tamaño-actividad; ni se conoce el funcionamiento cerebral en general, ni su correlación con las capacidades. No sabemos cuáles y cuántas de las diferencias de hoy son naturales o artificiales, ni si hay diferencias entre los sexos. Aunque lo artificial pudiese aislarse, nada nos permite afirmar que se revelaría lo natural o que podríamos acceder a ello (2010, 152; 156). Desconocemos aún las leyes de formación del carácter. Y en todo caso: el que estudia su naturaleza suele ser un hombre que se relaciona con la mujer a través de filtros y siempre desde la relación amo-esclavo $(2010,74)$. El sometimiento, la falta de libertad, el miedo, etc., impiden el conocimiento, la comunicación real y el trato humano. Se afirma que ciertas cualidades morales serían intrínsecas a las mujeres lo cual no son sino «estúpidos panegíricos», porque reconocen una superioridad moral a quienes se obliga a vivir sometidas; conceden que el poder masculino precisamente ha empeorado al hombre (2010, 173) y además los halagos coexisten con las descalificaciones. Se dice que comenten menos delitos o que tienen aversión a la guerra. Pero todo esclavo puede cometer pocos delitos y emprender pocas acciones. Se dice que tienden a la caridad, a la benevolencia y al proselitismo religioso. Para Mill, esto conduce en todo caso a una radicalización de animosidades religiosas en el propio país, y fuera es un objetivo ciego si no se considera nada más. La ética de la benevolencia y la caridad son perjudiciales si exoneran al beneficiario de su responsabilidad $(2010,195)$. La mujer también se dejaría llevar por sus sentimientos; sería proclive al favoritismo y al partidismo; no iría más allá del círculo más próximo siendo incapaz de universalismo moral (2010, 175). En fin, la naturaleza que se le supone es el «resultado de una forzada represión en algunas direcciones, y de un estímulo antinatural en otras (...) Ninguna otra clase de seres dependientes ha tenido su carácter tan enteramente distorsionado (...) como consecuencia de su relación con sus amos» $(2010,67)$. Ni siquiera ellas conocen sus capacidades: porque nunca se las requirieron (2010, 72). Sin embargo, para reconocer que las mujeres tienen los mismos derechos no se necesita determinar su naturaleza $(2010,78)$, basta con saber lo que ya se sabe: que son seres humanos.

19. «Auguste Comte intentó durante años disuadir a Mill (...). Para Comte, los resultados de la frenología confirmaban que las mujeres, como los niños, no poseían una estructura cerebral adecuada para realizar razonamientos complejos, ni para trascender el interés privado en beneficio del público» (Miguel Álvarez 190-191). Sobre Comte y Mill, ver: Guillin 2009. 
Suele esgrimirse también que ninguna ha llegado a ser un Homero, un Aristóteles, un Miguel Ángel o un Beethoven. Aunque todo lo que produce cada mente es necesariamente original al ser única, en todo caso a las obras de mujeres lo que les falta -remarca el autor- es una alta originalidad. Pero es que hasta ahora han sido obligadamente autodidactas partiendo generalmente de cero y de sí mismas, como si en cada campo no hubiese nada antes que ellas. Para producir algo de valía es imprescindible también la soledad ${ }^{20}$ y el tiempo: la mujer siempre tiene que estar al servicio de alguien, "generalmente de todo el mundo» (Mill 2010, 170), o si no entregada a la dictadura de resultar encantadora (Mill 2010, 168). Y no es menos necesaria cierta pasión: un ardiente deseo de celebridad, que en las mujeres se considera atrevimiento y un rasgo poco femenino. Por otro lado: «¿quién podrá saber cuántos de los pensamientos (...) fueron sugeridos por una mujer, habiendo sido la sola labor del hombre verificarlos y pulirlos? Si se me permite juzgar guiándome por mi propio caso, han sido, ciertamente, una gran proporción» (Mill 2010, $162)^{21}$.

Amin añade que la liberación de las mujeres no significa convertirse en hombres ni desempeñar por obligación las profesiones masculinas. Tienen capacidad para dedicarse a las profesiones liberales (2000b, 91), pero se permite señalar las más adecuadas: maestra y médica. Aduce en ambos casos cualidades tradicionalmente asignadas a las mujeres y, en el segundo, una consideración muy propia de la actitud que viene denunciando: «todos los hombres saben lo molesto que resulta tener enferma a una mujer de su familia, y verse necesitado de acudir a un médico varón, en especial cuando se trata de una dolencia específicamente femenina» (2000b, 90). En el caso de Mill, también afloran las reservas: «No pienso (...) que en un estado justo de cosas, sea una costumbre deseable el que la mujer contribuya con su trabajo a los ingresos de la familia» $(2010,118)$, aunque si tiene capacidades excepcionales podría trabajar fuera, siempre que su función como ama de casa no sufra menoscabo $(2010,120)$. En los ensayos de 1832 había escrito: «No es conveniente sobrecargar el mercado de trabajo con un doble número de competidores. (...) El marido debería poder ganar (...) todo lo necesario para los dos (...); redundaría en beneficio de la felicidad de los dos el que su ocupación

20. En Principios de economía política sostiene: «La soledad, en el sentido de estar solo, es esencial para cualquier meditación o carácter profundo» (Sánchez Fernández 17).

21. Diversos estudios corroboran -acudiendo también a la valiosa información epistolarque Harriet efectivamente está presente en la autoría y en la «maternidad» en varias de sus obras, una coautoría y colaboración -también con su hijastra- que Mill no duda en reconocer y alabar (Gil Ruiz 72-78; Guisán 100-102).

Feminismo/s 26, diciembre 2015, pp. 57-81 
fuera más bien adornar y embellecer la vida. Salvo en la clase obrera actual, ésta será su tarea diaria» $(2000,99)$.

\subsection{Matrimonio, poligamia y divorcio}

Amin explícitamente quería abordar la esfera privada porque era consciente de que aquí se escondía el último obstáculo para la emancipación. La mujer es conducida "por su padre, cual ganado, a un esposo (...) sin que ella tenga nada que decir» $(2000 \mathrm{~b}, 50)$. Su vida transcurre bajo el yugo de distintos hombres: «el del padre (...) el del marido después, y, a la muerte de este, bajo el de sus hijos o el de sus parientes varones o parientes de su esposo si no hubiera tenido hijos» $(2000 \mathrm{~b}, 33)$. Su condición se llama esclavitud porque «desde que nace hasta que muere no vive por sí ni para sí (...) Piensa con los pensamientos de su marido, por sus ojos mira y por sus oídos oye. Quiere lo que él desea, actúa según su dictado y ni tan siquiera se mueve a no ser que él se lo ordene. Es (...) un objeto, como un apéndice del hombre» (2000b, 51). Se espera que sea «una agradable mascota cuyas necesidades son cubiertas por su dueño a cambio de entretenimiento (2000a, 15).

En el caso de Stuart Mill, el matrimonio está en contradicción con los principios que rigen desde hace tiempo el mundo moderno: «ya no hay esclavos legales, excepto toda ama de casa» $(2010,179)$. Y al hilo de esta crítica, pone sobre la mesa un problema ausente en Amin: la ley aboca a la víctima de maltrato a volver con su maltratador $(2010,95)$. El matrimonio no debe ser una necesidad para asegurarse la manutención. La educación que le es inculcada a la mujer la hace, sin embargo, «no considerar como una degradación aquello que es la esencia de toda prostitución: el acto de entregar su persona por pan» $(2010,99)$. Paradójicamente, Mill no parece considerar la importancia de la emancipación económica, ni cómo evitar tal «prostitución», especialmente cuando propone que la mujer se quede en casa. Tanto él como Amin ponen de relieve, sin embargo, que en la familia como institución está el origen del problema de la pérdida de libertad de la mujer. Pero Mill relaciona matrimonio y reproducción de la especie, y hace de esta la causa última: para perpetuar el sistema «es necesario para la sociedad que las mujeres se casen y produzcan hijos; pero no lo harán a menos que se las obligue. Por lo tanto: es preciso obligarlas» (Mill 2010, 79). Y este hecho es independiente de toda religión, si bien para el autor egipcio las distintas tradiciones teológicas han sido un potente instrumento de legitimación.

Según Amin, la llegada del cristianismo no supuso un sistema que garantizase la libertad de las mujeres. El islam habría sido el primer sistema en estipular la igualdad entre los sexos $(2000 \mathrm{a}, 7)$ mejorando algunos aspectos de la 
situación de la mujer: igual capacidad legal para realizar adquisiciones, donar, tutelar y disponer de bienes, derechos todos ellos que en la época del autor todavía no habían sido logrados por todas las occidentales ${ }^{22}$. Regularizó también el matrimonio como institución, pero los teólogos lo definieron como «un contrato por el que un hombre tiene el derecho a dormir con una mujer» (2000a , 76), convirtiéndolo así en un medio por el que este puede controlar a un número de mujeres, simultánea o sucesivamente, para satisfacer su deseos sexuales. Sin embargo, el texto coránico $\left(\mathrm{XXX}, 21^{23}\right)$ se basa en el afecto y la bondad en la relación. La historia del islam ha sido también aquí contraria al ejemplo del Profeta y a los hadices que promueven el trato justo y afectuoso a las mujeres (2000a, 80).

La posición de Mill respecto al cristianismo es muy distinta, pese a sus críticas a la utilidad de la religión. Niega que el cristianismo haya legitimado la injusticia porque algo así sería contrario a su esencia (como si los hechos no pudiesen ir en contra de los principios, o como si el cristianismo fuese un ente que no puede ir en contra de su naturaleza). San Pablo habría tenido como objetivo propagar el cristianismo, no luchar contra las leyes existentes, lo cual no «debe interpretarse como una desaprobación de cualquier intento de mejorarlas en el momento oportuno» $(2010,115)$. El cristianismo es «la religión de la parte progresista de la humanidad», no de la parte decadente como el islam y otras ${ }^{24}$. Habría habido intentos de «convertirnos en una suerte de cristianos-musulmanes, con la Biblia por el Corán, prohibiéndonos toda clase de mejora (...) Pero esas gentes han sido resistidas, y la resistencia nos ha hecho lo que ahora somos, y nos hará lo que hemos de ser» (2010, 116).

Otro de los temas que aborda Amin es la poligamia, una práctica existente antes del islam. La considera un truco legalizado para que el hombre satisfaga sus deseos (2000a, 85). Es incompatible con la igualdad y la justicia y desprecia a la mujer. Ninguna la acepta de buena gana. El Corán autoriza un número de esposas, pero le exige al hombre una condición imposible de

22. Amin se refiere a la situación de las estadounidenses seguidas por las inglesas, las alemanas, las francesas, las austríacas, las italianas y las rusas (2000a, 7). Pero no precisa datos concretos.

23. «Y entre Sus signos está el haberos creado esposas nacidas de vosotros mismos, para que os sirvan de quietud, y el haber suscitado entre vosotros el afecto y la bondad. Ciertamente, hay en ello signos para gente que reflexiona».

24. Si bien no especifica cuáles y por qué, quizá pueda verse aquí tanto una influencia de la opinión de su padre como del contexto colonialista.

Feminismo/s 26, diciembre 2015, pp. 57-81 
cumplir: ser igual de justo con todas ellas ${ }^{25}$, lo cual debería entenderse como su prohibición. Amin rechaza también los supuestos legales con los que se justifica: la enfermedad y la esterilidad de la mujer, ya que esta no es culpable de su enfermedad y el deseo de descendencia no tiene por qué obligar a la poligamia, pues la primera mujer puede rechazar seguir como esposa. Tampoco acepta que la monogamia sea antinatural, sino un rasgo de evolución y progreso (Amin 2000a, 83). Concluye que la poligamia es una fuente de conflicto, perjudica la educación y el bienestar de los hijos y empobrece a las familias. Es un vestigio de sociedades primitivas en las que la mujer se consideraba una especie única situada entre el ser humano y los animales. Está condenada a desaparecer, entre otras cosas por la mayor concienciación y formación de los hombres.

En cuanto al divorcio, la posición de Amin es más combativa que la de Stuart Mill. Debería ser en todas las culturas parte integral del proceso del matrimonio. Solamente los cristianos lo prohibieron y esta prohibición religiosa ha continuado determinando las leyes de las sociedades occidentales y haciendo del matrimonio un contrato que solo puede disolver la muerte. El divorcio no debe estar regulado por ninguna religión. Es un acto legal y como tal debe celebrarse para que haya uniformidad y no se banalice el acto ni las consecuencias, ya que supone pérdida y adquisición de derechos y otros actos legales dependen de él (herencia, custodia de los hijos, manutención, etc.). Pero debe basarse en la justicia y la igualdad para que las mujeres puedan iniciarlo a voluntad.

\section{Más allá de los principios de la libertad y el interés común}

\subsection{La libertad y el interés común}

Según Amin, cuando nos enfrentamos a opiniones encontradas, como en el caso que no ocupa, debemos escoger lo que favorezca la libertad humana y el

25. «Si teméis no ser equitativos con los huérfanos, entonces, casaos con las mujeres que os gusten: dos, tres o cuatro. Pero, si teméis no obrar con justicia, entonces con una sola o con vuestras esclavas. Así, evitaréis mejor obrar el mal» (Corán IV, 3). Julio Cortés (78) explica en nota: «La primera parte de la aleya declara lícita la poligamia o, más precisamente, la poliginia. La segunda parte, arguyen algunos modernistas, prescribe implícitamente la monogamia, porque no hay hombre capaz de tratar con imparcialidad a sus esposas (...) La práctica islámica no lo ha entendido así. Probablemente hay que entender «huérfanas» (...) [Si teméis no ser justos casándoos con las huérfanas, pupilas vuestras, casaos con las mujeres no huérfanas que os gusten: dos, tres, cuatro]». Y la aleya 129: «No podréis ser justos [si sois polígamos] con vuestras mujeres, aún si lo deseáis. No seáis, pues, tan parciales que dejéis a una de ellas como en suspenso [en una situación ambigua: ni con marido ni sin él]». 
interés común (2000b, 28), un doble criterio que refleja tanto la influencia de Stuart Mill como de los principios del islam y del contexto reformista.

Según el criterio de la libertad, únicamente puede ser libre quien no somete su pensamiento, voluntad y acción a un yugo (Amin 2000b, 49). La mujer no será un ser completo a menos que su educación física y mental lo sea «considerando su dignidad personal (...) para que pueda ser feliz o desgraciada según su propia responsabilidad, y no de acuerdo con la de los demás» (Amin 2000b, 91). Garantizados todos sus derechos políticos -como se espera en Europa- se conseguirá la igualdad y el desarrollo de todo el potencial de las mujeres, que no son una simple máquina de reproducción (Amin 2000a, 73). Su liberación es, pues, un fin en sí misma. Pero la igualdad es solo el principio del camino al que Amin no quiere prescribir final ni forma concreta (2000a, 73).

El istislah o interés general es uno de los principios del derecho islámico. En la época de Amin es uno de los términos que especialmente Abdu y su seguidor Rashid Rida utilizarán para fundamentar la práctica del iytihad (esfuerzo de reflexión y análisis personal para extraer de las fuentes del derecho las respuestas que cada época y contexto requieren); la interpretación de la ley según el espíritu de esta (justicia y equidad) y el bien público, subrayando así la capacidad de evolución de la sharía y con ello la renovación del islam y del mundo árabo-islámico sin perder su identidad (Esposito 145) ${ }^{26}$. ¿Pero cómo es la transformación de las mujeres según el interés común? ¿Es compatible con el principio de la libertad y, sobre todo, con la caracterización de esta que acabamos de ver? La libertad está presente en el islam, si bien con las obvias limitaciones de un monoteísmo revelado. La cuestión es si este doble criterio puede articularse en el feminismo de Amin y si la religión no será en el fondo su particular «techo de cristal».

Pueden distinguirse dos sentidos del interés común en Amin: el de la humanidad y el del proyecto reformista-nacionalista. Según el primero, el fin de la civilización es «la marcha de la Humanidad por un solo camino (...) Eso es lo que queremos decir al hablar de evolucionar al ejemplo de los europeos y al señalar su imitación, indicando que volvemos la mirada a la mujer europea» (2000b, 138). La moral occidental no es condenable para Amin, pues los males de una sociedad son consecuencia natural de la existencia de la libertad. Y todo sistema tiene componentes perjudiciales, pues la absoluta perfección no es posible en esta vida $(2000$ a, 89$)$. Ha sido la diferencia de fe y la

26. Según Hj. Sartiyati, Qasim Amin se basaría en el texto de Abdu, «La prosperidad de la sociedad y el interés común» (21).

Feminismo/s 26, diciembre 2015, pp. 57-81 
enemistad entre Oriente y Occidente lo que ha ido generando imágenes falsas e irreales uno de otro, basadas en las pasiones y la ignorancia mutua (2000b, 141). Amin suscribe una concepción de Occidente no exenta de idealización, también respecto al tema de la liberación de la mujer ${ }^{27}$, pero no propone una imitación ciega. En primer lugar, porque aunque los logros de la humanidad tienen un lugar de origen, son patrimonio de esta, y pueden ser asumidos de forma activa por culturas e individuos. En Aforismos, su diario publicado póstumamente, rechaza precisamente la imitación ciega de sus compatriotas (Mazid 59). Egipto habría entrado en la etapa más importante de su historia porque camina hacia el progreso. Pero nunca se habría enfrentado a un peligro mayor: la expansión de un Occidente que se apropia sin escrúpulos de la riqueza y los recursos, una expansión que, sin embargo, no sería distinta a la del islam en su momento de esplendor. Pero ni antes ni ahora se trataría de guerras de religión ni de ideologías, sino de la lucha por la existencia: protección de las fronteras, ampliación de la zona de control e intereses comerciales e industriales (Amin 2000a, 66). La selección natural también es aplicable a las culturas y la fuerte vence a la débil (Amin 2000a, 63). El pueblo egipcio ha de plantar batalla con sus fuerzas intelectuales y educativas. En tercer lugar, porque aunque Occidente represente el bien asequible a la humanidad en esta etapa concreta, «siempre el anhelo del alma humana es mayor» (Amin 2000b, 149). Por eso, si se pregunta hacia dónde conduce la liberación de la mujer, es una incógnita que nadie puede despejar, de la misma forma que no sabemos qué pasará con el hombre (Amin 2000a, 119). En este sentido, el bien común sí es compatible con la libertad.

Amin había iniciado su reflexión sobre la mujer con la intención de centrarse en la esfera privada, pero pronto no puede mantener tal separación entre lo público y lo privado: los temas que denuncia están casi todos regulados por los códigos de estatuto personal, o sea, por las diferentes escuelas jurídicas. La situación política y familiar son «aspectos inseparables de todos los países y lugares y esto afecta a ambos sexos» (2000b, 34). Y la forma de gobierno influye en la vida privada y esta en el cuerpo social. Además, la esclavitud femenina a lo largo de la historia dista mucho de ser una cuestión privada. Por otra parte, y aquí está la cuestión, la liberación de la mujer es también un medio para un fin: el bien de Egipto y la renovación del islam, porque la familia sería la base y el motor del país, y la base de la familia es la mujer (Amin 2000a, 72). Y ello hasta el punto de que cada hombre adulto «es producto de

27. Por ejemplo, afirma: «Nadie entre ellos discute el derecho que la mujer tiene a disponer de su cuerpo y de su mente, excepto en lo que dictaminan las buenas costumbres» (2000b, 44), aunque no especifica qué entiende por «buenas costumbres». 
una mujer» (Amin 2000a, 71); ella es responsable de los defectos educativos de las generaciones e incluso del alto índice de mortalidad infantil. Su función es «la conformación de la moral comunitaria y de la ética, que influirán en la colectividad y decidirán el progreso (...) o su decadencia, más allá de toda suerte de constituciones, leyes y religiones» (Amin 2000b, 100). Pero una mujer velada, recluida e ignorante no tiene opiniones sobre negocios, movimientos políticos, arte, asuntos públicos o asuntos doctrinales; no tiene orgullo patriótico o sentimientos religiosos (Amin 2000b, 10). Un segundo aspecto del bien común de la nación se refiere al aprovechamiento del capital humano: si las mujeres no se forman, son una riqueza que no produce nada (Amin 2000a, 12). Si no pueden ganarse su sustento, son una carga social y unos parásitos que viven del esfuerzo de otros, una posición muy similar a la que ya defendiera Averroes $(2011,59)$ y que contrastaba con la actividad real de las clases humildes de ambas épocas.

En el caso de Mill, la liberación de las mujeres es de gran utilidad para la humanidad porque la ley del más fuerte no se superará mientras siga imperando en la familia $(2010,84)^{28}$. Debe practicarse y lograrse en la familia lo que se requiere para la sociedad (Mill 2010, 112), pero la responsabilidad no es exclusiva de la mujer, pues la otra escuela es la ciudadanía. La liberación de las mujeres enriquecería también la convivencia de los cónyuges al ser los dos seres humanos completos y formados. Y aumentaría «el fondo general de pensamiento y poder activo» (Mill 2010, 110), y la oferta de capacidades y habilidades en circulación y competición en la sociedad (aunque no se ve bien cómo, si la mujer no debe trabajar). Y lo más importante: la «felicidad privada para la mitad liberada de la especie humana» (Mill 2010, 110). Después de las necesidades más básicas, la libertad es la primera y más fuerte necesidad de la naturaleza humana, y no puede reprimirse sin consecuencias psicológicas como el resentimiento (Mill 2010, 110).

\section{2. "Volver a las raíces, a la gloria pasada. Ella nos iluminará»}

Según Amin, el islam no es ya la religión progresista e igualitaria cuyo impacto inicial en todos los países fue uno de los mayores motores de progreso y desarrollo. Y las causas han sido muchas: dejó de haber líderes competentes al frente de las naciones musulmanas ocupando otros pueblos el poder

28. La moral de la sumisión dejó paso a la caballerosidad, pero hoy es el tiempo de la justicia y el afecto mutuo. Los valores del honor caballeresco, «el punto culminante de la influencia de los sentimientos de las mujeres en el cultivo moral de la humanidad», han sido una fase intermedia pero insuficiente (Mill 2010, 109). 
(2000a, 66); desapareció al-Andalus y sus conocimientos fueron transferidos a Europa; la ignorancia generalizada acabó afectando a la forma de entender y vivir la religión; sus eruditos $(2000 \mathrm{a}, 65)^{29}$ promovieron el desprecio de las ciencias y los asuntos del mundo, debiendo el ser humano no buscar sus propios bienes ni elegir por sí mismo sino asumir el fatalismo. El islam se convirtió en un mosaico de costumbres y supersticiones inaceptables que fueron perpetuadas por gobiernos despóticos. Estos arrebataron a las estructuras políticas los mecanismos que regulaban los derechos y deberes del dirigente y de los ciudadanos, y que garantizaban que estos pudiesen exigir que se gobernase dentro de los límites de la sharía (2000a, 8).

Pero si el islam de hoy no es el genuino y la situación de la mujer musulmana ha sido y es contraria a este -una religión esencialmente progresista e igualitaria- la solución de Amin parece que es volver al pasado. De hecho, una de las grandes consignas de la época es recuperar el islam auténtico, el de los primeros tiempos: «volver a la raíces, a la gloria pasada. Esa época nos iluminará» (Amin 2000b, 129). Pero para Amin, el progreso viene inevitablemente acompañado de cambios según cada contexto (2000a, 5). El cambio es una constante en el universo dispuesto por Dios. La tradición no es una excepción, además de un conjunto de convenciones. Y Amin se pregunta por qué un musulmán debería creer que sus tradiciones no pueden ser cambiadas o reemplazadas por unas nuevas y que su deber es preservarlas para siempre. De hecho, los musulmanes no tienen el mismo estilo de vida, las mismas preferencias y tradiciones en todas las partes del mundo. Hay que rechazar la tradición «cuando (...) domina nuestra voluntad y nuestra capacidad de elección» (Amin 2000b, 129). La tradición suele controlar a un país más que ningún otro poder y es el componente menos proclive a cambiar. Las tradiciones son capaces incluso de reemplazar la religión existente, destruirla o transformarla hasta el punto de hacerla rechazable (Amin 2000b, 6). Exigir adaptarnos a la tradición es absurdo: como si un hombre que ha cambiado de talla, decidiese contraer su cuerpo en lugar de cambiar de traje (Amin 2000b, 102).

La gran enfermedad del mundo árabe es su dependencia del pasado. Ha sido inoculada por sus eruditos, la Universidad de al-Azhar, los «teóricos imaginarios» y los intelectuales que se presentan como críticos pero no dudan en buscar el apoyo de dictámenes y justificaciones religiosas. Los historiadores han falsificado el pasado y los alfaquíes han inventado hadices (Amin 2000b, 136). Entre todos han ido tejiendo un pasado ficticio y la convicción de que

29. «Its scholars and theologians, with the exception of few whose hearts have been illuminated by God, have manipulated it however they wanted, and in fact have made it an object of ridicule» (Amin 2000a, 65). 
en él se dio el islam genuino. Pero en ese pasado los gobiernos fueron manifestaciones personales de la voluntad arbitraria de los dirigentes; la situación familiar carecía de organización; no se produjo nada nuevo en el campo de la moral; el islam estuvo desgarrado por disensiones y en todas sus épocas hubo inmoralidad. Ese glorioso pasado empezó y acabó antes del inicio de la ciencia y la mentalidad modernas, por lo que necesariamente tuvo que adolecer de todas las deficiencias propias de ese estadio de la humanidad. Como no podía ser de otra manera, numerosos aspectos del pasado no tienen ya cabida en nuestro tiempo. La perfección humana «sólo la encontraríamos en un futuro muy lejano» (Amin 2000b, 136); nunca en el pasado de ninguna cultura. Por eso Amin se declara hereje $(2000 \mathrm{~b}, 4)$ del islam idealizado. Y denuncia la pereza mental que ha hecho de los musulmanes seres autosatisfechos y hostiles a toda nueva idea que vetan esgrimiendo, sin más, que es contraria al islam: "Nos hemos convertido en un suelo estéril, no apto para que crezca nada» $(2000 \mathrm{~b}, 4)$. A esta situación se suma el mal que arrastra la humanidad: el dominio ejercido sobre las mujeres, un hecho independiente de culturas y credos. No hay más que ver la similitud de su situación en todo el mundo y cómo los estereotipos sobre la mujer son transculturales ${ }^{30}$. El reformismo es un movimiento histórico y la reforma (islah) ${ }^{31}$ es una constante del islam, pero para Amin es ante todo una categoría antropológica que divide a la humanidad (2000b, 44). Reformista es quien aparta de sí los factores que influyen en el desarrollo de sus sentimientos, deja a un lado la herencia recibida, analiza las cuestiones objetivamente desde todos sus puntos de vista y atiende a los datos empíricos, a la opinión y reflexión personal y a la verdad (Amin 2000b, 47).

\section{3. «Hasta que las mujeres mismas hayan contado todo lo que tienen que contar»}

Según Mill, los hombres limitan a las mujeres en la esfera pública para mantener su subordinación en la privada, porque el sexo masculino «no puede tolerar la idea de vivir con un igual» $(2010,121)$. El triunfo de la ley del más fuerte apunta, pues, a un problema en la constitución de la identidad masculina, pero puede afectar a todas las relaciones humanas y esto es lo que Mill también pone de relieve. Decíamos más arriba que la naturaleza

30. Poco piadosa, escasamente inteligente, elemento de discordia, trampa del maligno, creada para satisfacer al varón y refractaria al conocimiento (Amin 2000b, 25).

31. Tariq Ramadán especifica que implica llevar aquello de lo que se trate a su estado original, siendo este último el momento en que era como debía ser, no había degenerado. Implica pues, mejorar, reformar, curar hasta volver a dicho estado (2009).

Feminismo/s 26, diciembre 2015, pp. 57-81 
de una mujer llega a ser una incógnita incluso para sí misma. Su identidad será opaca «hasta que las mujeres mismas hayan contado todo lo que tienen que contar». Mill se refiere en más de una ocasión a las posibilidades de la literatura al respecto. En "¿Qué es la poesía?»" no se pronunciaba sobre cuestiones feministas, pero destacaba la poesía frente a la novela en un sentido revelador: «la descripción de las operaciones de la emoción humana más profundas y secretas, interesa únicamente a quienes les recuerda lo que han sentido, o les despierta la imaginación para evocar lo que podrían sentir, o lo que podrían haber sentido si sus circunstancias externas hubieran sido otras» (Mill 2002, 39). La poesía exige la total inconsciencia del poeta en cuanto a la presencia de un oyente, y no es posible tampoco si el autor «no puede conseguir eliminar de su obra el rastro de estas miradas al cotidiano mundo exterior, ni puede expresar sus emociones exactamente igual que las ha sentido en soledad, o como cree que debería sentirlas, aun cuando fueran a permanecer eternamente silenciadas» (Mill 2002, 44). Por tanto, si la literatura es un espacio de liberación para la mujer, la poesía parece uno muy especial, aunque sus condiciones de posibilidad revelan un problema de gran importancia para el tema que nos ocupa: hará falta esa liberación del mundo exterior y de la presencia de un particular oyente. El universo simbólico masculino aún debe, pues, ser derruido también en el interior. El colonizador ha de ser expulsado del sentimiento, del pensamiento y de la acción. También aquí, en el caso de Mill, hay que ir más allá de los principios de libertad y del bien común.

\section{Conclusiones: esperando el «momento oportuno»}

Amin denuncia una serie de situaciones concretas de la esclavitud de la mujer que, como es lógico por las diferencias de contexto cultural, no todas coinciden con las que cuestionó Mill. Para este la razón de fondo del sometimiento de la mujer es la persistencia de la ley del más fuerte en las relaciones humanas. Mill milita activamente en la consecución del derecho al voto de las mujeres, si bien no parece ser consciente de la necesidad de liberación económica. Un aspecto importante de su reflexión es la necesidad de otro tipo de liberación, más interna y personal, que ejemplifica con el recurso a la literatura, un aspecto que no suele abordarse de su filosofía. Para Qasim Amin,

32. «desilusionado con la constricción intelectual de la teoría [utilitarismo], fue matizando su posición hasta llegar a incluir en la palabra 'utilidad' los placeres de la imaginación y de las emociones más elevadas, coincidiendo en esto con los escritores románticos (Sánchez Fernández 15-16). 
el caballo de batalla también es la ley del más fuerte y también defiende el derecho al voto, pero la abolición de la ley del más fuerte no es independiente de la superación de la enfermedad del pasado y de una reforma teológica del islam para que siga su espíritu progresista de los primeros tiempos y el del propio Corán. En ambos autores, sin embargo, los principios de la libertad y el bien común entran en conflicto en algunos asuntos concretos, aunque en Amin es muy significativa su insistencia en la indeterminación futura de la imagen de la mujer.

Qasim Amin dejó constancia en sus memorias de la necesidad de pronunciarse y actuar ante la urgencia de los hechos: «si los reformadores tuviesen que esperar a la aprobación de la opinión pública, el mundo sería el mismo que en la época de Adán y Eva» (2000a, xiii). En la obra publicada especifica que su objetivo es poner sobre la mesa una cuestión que muchos intelectuales han pasado por alto, no llevar a cabo un examen exhaustivo, y aún menos dilucidar cuestiones metafísicas, en el sentido de una filosofía sistemática. Quiere plantar una semilla cuyos brotes aparecerán en la mente de la generación futura, la habitante de la verdadera al-madina al-fadila (la ciudad ideal), podemos decir nosotros, siguiendo su imagen farabiana. Dicha urgencia afecta, sin embargo, al nivel de su discurso, que a veces parece más un panfleto. Y puede también que las contradicciones que se aprecian se expliquen en parte por ello, además de porque él mismo forma parte de la mentalidad y del contexto a superar. Tampoco hay que descartar la censura ni la autocensura. Y algo más: el tema en cuestión, que aborda prejuicios, costumbres y afectos, supera un planteamiento basado en una mera refutación conceptual como Mill pone de relieve $(2010,33)$.

De cualquier modo, Amin utiliza una batería de argumentos: laicos, ilustrados, religiosos, reformistas, nacionalistas y no acaba de desprenderse de la religión, pero quizá por eso mismo su mediación sea hoy, en ciertos aspectos, más afín a la realidad de los retos de nuestro tiempo en un mundo a varias velocidades, y más útil e inspiradora para el diálogo interreligioso. Aplicándole la misma regla hermenéutica que él adopta respecto al pasado y a la tradición, es evidente que de su propuesta no es posible recuperarlo todo. Pero si los antiguos son dignos de imitar porque «instauran la reflexión y la discriminación como método», uno de los aspectos más interesantes del pensamiento de nuestro autor es su valentía a la hora de opinar abiertamente sobre la cuestión del velo, apuntando con sus tesis más allá del feminismo de la igualdad e incluso del feminismo. Por otro lado, al optar por el espíritu ético de la religión y dar visibilidad a los hadices y a los ejemplos de mujeres, lleva a cabo un significativo ejercicio de justicia histórica, poniendo de paso 
las bases para una islamología feminista y una «sharía liberal» (Amin 2000a, 7). Mill no hace de la religión el fundamento de ningún aspecto de su propuesta, pero se echa en falta una crítica a la religión desde la perspectiva de la liberación de las mujeres ${ }^{33}$. No menos importante es la propuesta de Amin de abordar de forma crítica el pasado, asumir activamente el patrimonio de la humanidad, adoptar el reformismo como actitud, o el aprendizaje de la libertad. Al contrario de lo que pudiera parecer, los oprimidos no son per se los más proclives a la justicia y a la compasión, ni los más amantes de la libertad. La única planta que crece en una nación oprimida es la depravación (Amin 2000a, 9): se respeta solo la fuerza y disuade solo el miedo. No somos déspotas -se queja- pero creemos que la única forma de mantener la estabilidad familiar es la tiranía (Amin 2000b, 66). Egipto ha vivido políticamente en la esclavitud y aún no sabe qué hacer con la libertad (Amin 2000a, 73). Tanto él como Mill muestran que los principios de la libertad y el bien común deben reelaborarse sin perder de vista, en un caso, una patología cultural como es el lastre del pasado; en otro, la liberación interior para la construcción de un nuevo orden simbólico, una cuestión que da lugar en Mill a una interesante lectura de la poética. Muestran también cómo el problema de la desigualdad entre los sexos, va más allá de la ley del más fuerte y no tiene su origen en las religiones: es transcultural y debe combatirse en la construcción de la identidad masculina y al nivel de la educación de los afectos.

Pesa, sin embargo, ese «momento oportuno» del que habla Amin y que también suscribe Mill en gran medida (al menos respecto a su juicio sobre el cristianismo). Paradójicamente, Amin acaba posponiendo sin fecha los cambios (2000a, 60). La mujer egipcia «no está preparada (...) necesita tiempo» (2000b, 76). El argumento del «momento oportuno», por cierto, no dejará de esgrimirse en otros contextos, como ya parecía vislumbrar -entre el dolor y la rabia- Olympe de Gouges: «¡Oh, mujeres!, ¡mujeres!, ¿cuándo dejaréis de estar ciegas?, ¿qué ventajas habéis obtenido de la revolución?: un desprecio más marcado, un desdén más visible». Por suerte, ese momento no tiene

33. En La utilidad de la religión la perspectiva de género se reduce a afirmar que, según la Biblia, la obligación religiosa no es más estricta con las mujeres en el caso de las relaciones sexuales ilícitas, recogiendo el ejemplo citado por Bentham (el de las relaciones sexuales ilícitas). El deber religioso, cuando no está reforzado por el poder de la opinión pública, no sería muy efectivo por sí mismo en la conducta, según Mill (2012: 91). ¿Quiere decir esto que la Biblia es más benévola con las relaciones ilícitas que la presión social? El tema al que se está refiriendo aquí Mill no deja de tener relación con esa defensa de la libertad que emprende en su célebre escrito, pero tampoco pasan desapercibidas las connotaciones personales. 
dueño. Mill apuntó las consecuencias psíquicas negativas de la represión de la libertad individual de las mujeres, pero más bien respecto a las relaciones entre los sexos $(2010,213)$. Pero Qasim Amin se pronuncia de forma más rotunda con una imagen producto de su propia experiencia: hay pensamientos -y el de la liberación de las mujeres sería uno de ellos- con una fuerza tal que superan cualquier duda en quien los alcanza, ya sea sobre la posibilidad de comunicarlos o sobre sus consecuencias. Si el individuo no utiliza esa fuerza para ponerlo en práctica, si insiste en combatirlo, en coartarlo o reprimirlo, se volverá contra él como un gas que no puede ser contenido bajo presión.

\section{Referencias bibliográficas}

ABISAAB, Malek y Rula Jurdi Abisaab. «A Century After Qasim Amin: Fictive Kinship and Historical Uses of «Tahrir al-Mara». Al-Jadid 6. Núm. 32 (2000). http://www.aljadid.com/content/century-after-qasim-amin-fictive-kinshipand-historical-uses-tahrir-al-mara, consultado el 31.05.2014.

AmIn, Qasim. The Liberation of Women. The New Woman. Two Documents in the History of Egyptian Feminism. Ed. Samiha Sidhom Petersom. The American University in Cairo Press, 2000a.

Amin, Qasim. La nueva mujer. Instituto Egipcio de Estudios Islámicos. Madrid, $2000 \mathrm{~b}$.

AmIn, Qasim. Tahrir al-mar'ah. Facsimile Publisher. Reprints from the collection of the University of Michigan Library, 2015a.

AmIN, Qasim. Al-Mar'ah al-jadidah. Facsimile Publisher. Reprints from the collection of the University of Michigan Library, 2015b.

Amorós, Celia, y Ana de Miguel (eds.). Teoría feminista: de la Ilustración a la globalización. 1. De la Ilustración al segundo sexo. Madrid: Minerva Ediciones, 2007.

Amorós, Celia. Vetas de Ilustración. Reflexiones sobre feminismo e Islam. Madrid: Cátedra, 2009.

Averroes. Exposición de la 'República' de Platón. Madrid: Tecnos, 2011.

AZAOla PIAZZA, Bárbara. «Participación política y social de la mujer egipcia». Feminismo/s 3 (2004): 161-174.

BERKEY, Jonathan Porter, The Transmission of Knowledge in Medieval Cairo. Princeton University Press, 1992.

El Corán. Edición, tradición y notas de Julio Cortés. Barcelona: Herder, 2007.

Espósito, John L., y Natan DeLong-Bas. Women in Muslim Family Law. Syracuse University Press, 2011.

GIL RuIz, Juana María. «El debate de John Stuart Mill y Harriet Taylor en los Ensayos sobre la igualdad sexual». John Stuart Mill y las fronteras del liberalismo. 
Coord. Manuel Escamilla Castillo. Granada: Universidad de Granada, 2004, 65-97.

Guillin, Vincent. Studies in the History of Political Thought, Volume 1: Auguste Comte and John Stuart Mill on Sexual Equality: Historical, Methodological and Philosophical Issues. Boston, MA, USA: BRILL, 2009.

GUISÁN, Esperanza. «Harriet Taylor Mill, John Stuart Mill y la ética del siglo XXI». John Stuart Mill y las fronteras del liberalismo. Coord. Manuel Escamilla Castillo. Granada: Universidad de Granada, 2004, 99-121.

KHURI-MAKDISI, Ilham. The Eastern Mediterranean and the Making of Global Radicalism, 1860-1914. Berkeley: University of California Press, 2010.

MARín, Manuela. «Women, gender and sexuality». The New Cambridge History of Islam. Vol. 4, Islamic Cultures and Societies to the End of the Eighteenth Century. Ed. Robert Irwin. Cambridge University Press, New York, 2010, 355-379.

MASSAD, Joseph A. Desiring Arabs. University of Chicago Press, 2007.

MAZID, Nergis. «Western Mimicry or Cultural Hybridity: Deconstructing Qasim Amin's «Colonized Voice». American Journal of Islamic Social Sciences 19 (2002): 42-67.

Miguel Álvarez, Ana de. «El feminismo en clave utilitarista ilustrada: John S. Mill y Harriet Taylor Mill». Teoría feminista: de la Ilustración a la globalización. 1: De la Ilustración al segundo sexo. Eds. Celia Amorós, Ana de Miguel. Madrid: Minerva Ediciones, 2007, 175-210.

MiLL, John Stuart. «¿Qué es la poesía?». John Stuart Mill, Thomas Love Peacock, Percy Bysshe Shelley. El valor de la poesía. Ed., trad. y notas Eduardo Sánchez Fernández. Madrid: Hiperión, 2002.

Mill, John Stuart \& Taylor Mill, Harriet. Ensayos sobre igualdad de los sexos. Madrid: Antonio Machado Libros, 2000.

MiLL, John Stuart. El sometimiento de la mujer. Madrid: Alianza Editorial, 2010.

MiLL, John Stuart. Sobre la libertad. Madrid: Alianza Editorial, 2013.

MiLl, John Stuart. El utilitarismo. Madrid: Alianza Editorial, 2014.

MiLl, John Stuart. Tres ensayos sobre la religión. Madrid: Tecnos, 2012.

MiraUt MARTín, Laura. «Los derechos de la mujer en el feminismo moderado de John Stuart Mill». Anuario de filosofía del derecho 23 (2006): 101-130.

MiYARES, Alicia. «El sufragismo». Teoría feminista: de la Ilustración a la globalización. 1: De la Ilustración al segundo sexo. Eds. Celia Amorós, Ana de Miguel. Madrid: Minerva Ediciones, 2007, 245-293.

NooH, Ramadan. Anfänge der Frauenbewegung und Genderdiskussion in Ägypten. Am Beispiel von Qasim Amin und Hoda Sha'arawi als Begründer, GRIN Verlag, 2014.

PATEL, Abdulrazzak, The Arab Nahdah. The making of the Intellectual and Humanist Movement. Edinburgh University Press, 2013. 
RAMADÁN, T. Radical Reform. Islamic Ethics and Liberation. New York, Oxford University Press, 2009.

ReID, Donald M. The Odiyssey of Farah Antun. A Syrian Christian's Quest for Secularism. Minneapolis \& Chicago: Bibliotheca Islamica, 1975.

REID, Donald M. Cairo University and the making of modern Egypt. Cambridge University Press, 2002.

Robinson, Francis. «Education». The New Cambridge History of Islam. Vol. 4. Islamic Cultures and Societies to the End of the Eighteenth Century. Ed. Robert Irwin. Cambridge University Press, New York, 2010, 497-531.

Rossi, Alice B. «Sentimiento e intelecto. La historia de John Stuart Mill y Harriet Taylor Mill», en Stuart Mill, John \& Taylor Mill, Harriet, Ensayos sobre igualdad de los sexos. Madrid: Antonio Machado Libros, 2000, 21-87.

RUIz DE ALMODÓvar, Caridad. Historia del movimiento feminista egipcio. Granada: Universidad de Granada, 1989.

SAINT-PROT, Charles. La tradición islámica de la reforma. Barcelona: Bellaterra, 2014.

SHAnley, Mary L. «The subjection of women». The Cambridge Companion to Mill. Ed. John Skorupski. Cambridge University Press, 1998, 396-422.

SARTiYATI, H.J. «Konsep Emansipasi wanita Menurut Qasim Amin». At-ta'lim 3 (2012): 19-25.

Viviani, Paola. Un maestro del Novecento arabo. Farah Antun. Roma: Jouvence Editoriale, 2004.

ZACHS FRUMA, y Sharon halevi. Gendering culture in Greater Syria. Intellectuals and Ideology in the Late Ottoman Period. London, New York: I.B. Tauris, 2015. 